\title{
Prothrombotic fibrin network characteristics in patients with acromegaly: a novel mechanism for vascular complications
}

\section{Nikolaos Kyriakakis ${ }^{1,2}$, Nikoletta Pechlivani' ${ }^{2}$, Julie Lynch', Natalie Oxley², Fladia Phoenix ${ }^{2}$, Khyatisha Seejore ${ }^{1,2}$, Steve M Orme', Ramzi Ajjan ${ }^{2}$ and Robert D Murray ${ }^{1,2}$}

${ }^{1}$ Department of Endocrinology, Leeds Centre for Diabetes and Endocrinology, St James's University Hospital, Leeds Teaching Hospitals NHS Trust, Leeds, UK and 'Division of Cardiovascular and Diabetes Research, Leeds Institute of Cardiovascular and Metabolic Medicine (LICAMM), University of Leeds, Leeds, UK
Correspondence should be addressed to R D Murray

Email

robertmurray@nhs.net

\begin{abstract}
Objective: There remains increased cardiovascular mortality in patients with acromegaly. This study aims to evaluate whether GH/IGF-1 excess increases vascular disease by adversely affecting fibrin network characteristics.

Design: Cross-sectional study in 40 patients with acromegaly (21 males, age $53 \pm 13$ years) and 40 age/gender-matched controls.

Methods: Clot structure was analysed using a validated turbidimetric assay and fibrin networks were visualised by laser scanning confocal microscopy (LSCM). Metabolic profile parameters, body composition, plasma fibrinogen and PAI-1 were also assessed.

Results: Twenty-two patients had active acromegaly and 18 were in remission. There was no difference in qualitative patient characteristics between the two groups. Both groups had less favourable body composition and cardiovascular risk profile compared with controls. Despite no difference in clot formation and lysis parameters between the two patient groups, active disease patients had higher fibrinogen and clot maximum absorbance compared with controls, after adjusting for BMI $(3.8 \pm 0.2$ vs $2.6 \pm 0.2 \mathrm{mg} / \mathrm{mL}, P<0.001$; and $0.39 \pm 0.02$ vs $0.33 \pm 0.01$ arbitrary units, $P=0.03$, respectively). Patients in remission had higher fibrinogen compared with controls following adjustment for BMI $(3.3 \pm 0.2$ vs $2.6 \pm 0.2 \mathrm{mg} / \mathrm{mL}, P=0.02)$ but not clot maximum absorbance $(0.35 \pm 0.03$ vs $0.33 \pm 0.02$ arbitrary units, $P=0.6$ ). LSCM showed increased fibrin network density only in active disease patients, consistent with turbidimetric analysis. In addition to active disease, BMI, fat mass and skinfold thickness were associated with higher clot density and longer lysis time.

Conclusions: Patients with active acromegaly have more compact clots, thus conferring increased thrombosis risk. Prothrombotic fibrin networks may represent one mechanism for enhanced vascular risk in active acromegaly.
\end{abstract}

\section{Introduction}

Acromegaly has been associated with increased overall mortality compared with the general population. Two meta-analyses published in 2008 showed a mean standardised mortality ratio (SMR) of 1.72 (1) and 1.70 (2), respectively. However, overall mortality rates in acromegaly have been reducing with time, reflecting advancements in therapeutic interventions and the higher remission rates with modern treatments. This is reflected in a more recent meta-analysis from 2018, in which the SMR from clinical studies published after 2008 
is not significantly higher compared with the general population, whereas studies published before 2008 demonstrated an increased SMR at 1.76 (3).

In contrast to overall mortality, there resides an excess mortality related to increased cardiovascular and cerebrovascular disease $(3,4,5,6,7)$. In the recent metaanalysis, the SMR for cardiovascular death was higher in acromegaly in studies published both before (SMR 2.38) and after 2008 (SMR 1.67), with similar findings for cerebrovascular disease (3). Additionally, cardiovascular mortality increases significantly with GH levels $>2 \mu \mathrm{g} / \mathrm{L}$ and elevated IGF-1 (>2 standard deviation scores) (5).

Abnormalities of coagulation and fibrinolysis have been considered to contribute to the increased cardiovascular risk in acromegaly. Elevated levels of plasma fibrinogen have been consistently reported in several studies, particularly in patients with active disease $(8,9,10,11,12,13,14,15)$. Data regarding other markers of coagulation and fibrinolysis are scarce and often conflicting, and studies have been limited by small number of participants. Findings include elevated antithrombin III, tissue plasminogen activator (t-PA) and plasminogen activator inhibitor 1 (PAI-1) $(12,16)$. In contrast, other studies have reported no difference in PAI-1 and t-PA levels between patients with acromegaly and controls $(8,9)$. Lower levels of proteins $\mathrm{C}$ and $\mathrm{S}$ (which have an inhibitory effect on the coagulation cascade) have been found in patients with active acromegaly compared with healthy controls (14) and with patients with disease control (11). A common caveat in these studies is the focus on a single coagulation factor, which gives an incomplete picture of the thrombotic risk. A more comprehensive marker of thrombotic environment is fibrin network structure and susceptibility to lysis. This can be studied using a validated turbidimetric assay as previously shown $(17,18)$. The advantage of this technique is that it takes into account quantitative and qualitative changes in a large number of coagulation proteins, consequently translating the findings into alterations in fibrin clot properties. Recent work has shown that fibrin clot characteristics can predict adverse vascular outcomes in individuals sustaining a cardiac event, even after correction for a large number of clinical and biochemical vascular markers (19).

Establishing how elevated GH levels translate into increased vascular morbidity and mortality remains elusive. We hypothesise that one mechanism through which disturbances of the GH/IGF-I system increase vascular disease is by the induction of prothrombotic fibrin networks. We therefore tested this hypothesis in a cross-sectional pilot study evaluating properties of clot formation and lysis in a population of patients with acromegaly.

\section{Subjects and methods}

\section{Participants' recruitment}

In this cross-sectional pilot study, 40 consecutive patients with acromegaly were recruited. Acromegaly had been diagnosed in all patients prior to the recruitment to the study, by failure to suppress $\mathrm{GH}$ to $<0.3 \mu \mathrm{g} / \mathrm{L}$, as measured by a two-site chemiluminescent immunometric human GH assay, during a 2-h oral glucose tolerance test, with a $75 \mathrm{~g}$ of oral glucose load. Patients were approached when attending for clinic appointments. Additionally, age and sex-matched healthy individuals were recruited from patients' relatives and staff members of the Leeds Teaching Hospitals (via advertisement material displayed in outpatient clinic areas and circulated via electronic mail) to provide control data. Exclusion criteria included history of known haematological disorder predisposing to a thrombotic or bleeding tendency; existing treatment with antiplatelet or anticoagulant medications; patients with past or present malignant disorders and individuals unable to provide informed consent. The study was approved by the North West - Greater Manchester West Research Ethics Committee (Reference ID: 15/NW/0400). Informed consent was obtained from all study participants.

Based on the American Endocrine Society clinical practice guidelines of 2014 (20), patients were divided into two groups: patients with disease remission $(\mathrm{GH}<1 \mu \mathrm{g} / \mathrm{L}$ and IGF-1 within the age-specific reference range) and patients with active acromegaly (GH $>1 \mu \mathrm{g} / \mathrm{L}$ and IGF-1 above the reference range) or dichotomous GH/IGF-1 results $(\mathrm{GH}<1 \mu \mathrm{g} / \mathrm{L}$ and $\mathrm{IGF}-1$ above the reference range or $\mathrm{GH}>1 \mu \mathrm{g} / \mathrm{L}$ and IGF-1 within the reference range).

\section{Study outcomes}

The primary outcome was to evaluate clot structure properties in patients with acromegaly, exploring the effect of disease activity on clot structure properties and compare these with controls. Secondary outcomes included measuring key components of clot formation (fibrinogen) and lysis (PAI-1), while assessing for conventional surrogates of cardiovascular risk (lipid profile, body composition, glucose profile and prevalence of metabolic comorbidities) and $\mathrm{C}$ reactive protein (CRP), a marker of inflammation which has been associated with coronary artery disease (21). 


\section{Anthropometric assessment}

Evaluation included measurement of weight, height, waist and hip circumference, skinfold thickness at bicep, tricep, infrascapular and suprailiac areas and body composition by bioelectrical impedance (Tanita TBF300MA, Middlesex, UK).

\section{Sample collection}

Blood samples were obtained in the morning (0800-1000 h) following an overnight fast. The first $10 \mathrm{~mL}$ of blood were used for clinical laboratory investigations (lipid profile, fasting glucose and $\mathrm{HbA} 1 \mathrm{c}$ ), and, additionally, anterior pituitary hormone profile for the patient group, including random GH and IGF-1. A further $20 \mathrm{~mL}$ blood sample was collected into a citrate tube, centrifuged within $2 \mathrm{~h}$ upon collection and the plasma stored at $-80^{\circ} \mathrm{C}$ until analysis. All blood samples were obtained without applying a tourniquet.

\section{Clot structure analysis}

Turbidimetric analysis was used to analyse fibrin polymerisation characteristics in the clots formed ex vivo and to study fibrinolysis speed. Plasma samples were treated with thrombin and calcium using a microtiter plate spectrophotometer and changes in optical density were measured $(22,23)$. Rates of fibrinolysis were analysed in the presence of tPA, both at the beginning of the clotting reaction and after formation of the mature clot. A number of clot structure parameters were studied including:

(i) Maximum absorbance (MA): measure of fibrin network density and fibre thickness. It has previously been shown that higher MA is associated with increased cardiovascular risk $(17,22,24)$.

(ii) Lag time: the time required from the start of the reaction to the beginning of clot formation. Shorter duration of lag time has been associated with increased thrombotic potential (17).

(iii) Lysis time: the time required for the clot to reach 50\% lysis. Longer lysis time indicates increased resistance to fibrinolysis, which is associated with increased cardiovascular risk $(18,25)$.

(iv) Lysis area: a complex measure of clot formation and lysis. Larger lysis area is associated with increased cardiovascular risk (22).

\section{Laser scanning confocal microscopy (LSCM)}

Two pooled plasmas were produced; one of the patients with active acromegaly $(n=22)$ and a second of the patients with disease remission $(n=18)$. The pooled plasmas from each patient group were compared with controls, as well as with each other. Fibrin clots from these pooled samples were visualised using confocal microscopy.

Fibrin clots were created by diluting $7.5 \mu \mathrm{L}$ from each pooled plasma with $20.4 \mu \mathrm{L}$ of permeation buffer with the addition of Alexa 488-labelled fibrinogen at approximately 5\% (0.105 M) (Thermo Fisher Scientific/Life Technologies) for $30 \mathrm{~min}$ at ambient temperature. Following incubation, activation mix consisting of $0.05 \mathrm{U} / \mathrm{mL}$ human thrombin (Merck Chemicals Ltd, Nottingham, UK) and $5 \mathrm{mM} / \mathrm{L}$ $\mathrm{CaCl}_{2}$ in permeation buffer was added. The mixture was loaded to a $15-\mu \mathrm{L}$ Ibidi (Applied Biophysics, Troy, NY) slide in duplicate to a well. The clots were visualised using a LSM880 microscope (Carl Zeiss) using $40 \times 1.4$ oil objective lens. Three $\mathrm{Z}$ stacks of each clot were taken, with a range of $20.3 \mu \mathrm{m}$ at intervals of $0.7 \mu \mathrm{m}$ (total of 30 slices). The number of fibres per $100 \mu \mathrm{m}$ was calculated in each stack using ImageJ® software. The average number from the three stacks was determined in each study group to represent the density of the clot fibrin network.

\section{Laboratory assays}

Fibrinogen was measured using the Clauss method (26), while PAI-1 and CRP were measured by commercial ELISAs as per manufacturer's protocols (Thermo Fisher ${ }^{\circledR}$ Human PAI-1 Platinum ELISA BMS2033 and ab99995 C Reactive Protein (CRP) Human ELISA Kit, respectively). GH, IGF-1 and SHBG were measured using Siemens Immulite 2000 (GH calibrated against WHO NIBSC IS 98/574). Total cholesterol, HDL cholesterol and triglycerides were measures by the ADVIA Chemistry Cholesterol Concentrated assay, ADVIA Chemistry Direct HDL Cholesterol and ADVIA Chemistry Triglycerides_2 Concentrated assay, respectively, while LDL cholesterol was calculated using the Freidewald equation. Serum glucose was measured by an enzymatic assay based on the method by Slein, using hexokinase and glucose-6phosphate dehydrogenase enzymes. HbA1c was measured by the Tosoh G8 HPLC Analyzer, which utilises the IonExchange method. All assays were performed in the routine clinical biochemistry laboratories within the Leeds Teaching Hospitals and have been regularly validated by internal quality control and external quality assessment. 


\section{Statistical analysis}

Descriptive data are presented as mean and s.D., or median and interquartile range for parametric and non-parametric data, respectively. Non-paired $t$-test for continuous variables and Mann-Whitney $U$-test (for variables which failed normality test) were used to assess the difference in the values between different comparison patient groups. The Chi-square or Fisher Exact test was used to compare proportions between the different study groups. Comparisons in the clot structure properties between patients and controls were performed adjusting for BMI, using univariate analysis of covariance test (ANCOVA).

Multiple linear regression analysis was also performed. The models used included lag time, clot MA, lysis time, lysis area, fibrinogen and PAI-1 as dependent values. For the patient group, independent values included patient's age at the time of the study; gender; BMI, or fat mass, or waist/hip ratio, or summative skinfold thickness; GH or IGF-1 at the time of the study; use of GH/IGF-1 lowering medications at the time of the study; history of diabetes or impaired glucose tolerance (IGT); history of hypertension; dyslipidaemia; smoking status; duration of active disease and duration of disease remission. Fibrinogen was also included as an independent value in the regression models, which had lag time and clot MA as dependent values. PAI-1 was an independent value in the regression models in which lysis time and lysis area were tested as dependent values. For the control group, independent values included age; gender; BMI, or fat mass, or waist/hip ratio, or summative skinfold thickness; smoking status and levels of HbA1c, LDL and HDL cholesterol.

A $P$ value of $<0.05$ was considered statistically significant. Statistical analysis was performed using the statistics software 'SigmaPlot'.

\section{Results}

\section{Participants' characteristics}

A total of 91 patients with a history of acromegaly were screened for this study. Twenty-one patients were excluded as they were on treatment with antiplatelet or anticoagulant agents (four patients due to previous venous thromboembolic event; eight for secondary prevention due to previous vascular disease; three for thromboprophylaxis due to atrial fibrillation; one due to metallic heart valve; four patients for primary prevention due other additional cardiovascular risk factors and one was on aspirin without a clear indication). Additionally, one patient was excluded due to myelodysplastic syndrome.

Thirty patients were excluded from the study for other reasons: 16 were unable to attend for the study visit due to personal/social reasons; two patients had developed GH deficiency following treatment for acromegaly (confirmed by dynamic pituitary test); one patient was undergoing chemotherapy for bowel cancer at the time of recruitment; two were unable to provide informed consent due to cognitive impairment and language barrier and nine patients declined to participate without declaring any specific reasons.

Forty patients with acromegaly were recruited to the study; $55 \%$ of patients $(n=22)$ had active acromegaly or dichotomous GH/IGF-1 results at the time of recruitment to the study, who for the purpose of this study are referred as active disease group (or Group 1); and $45 \%$ of patients $(n=18)$ were in remission (Group 2). Table 1 summarises patients' clinical characteristics and acromegaly-related medical history.

Forty healthy volunteers matched for age and sex with patients were recruited. Four controls (10\%) were already established on treatment for hypertension with reasonable blood pressure control (BP range at the time of the study $129 / 87-143 / 89 \mathrm{mmHg}$ ); one was on atorvastatin for primary prevention; one was on stable dose of levothyroxine for primary hypothyroidism; one was on a progesterone implant and one on female hormone replacement for menopausal symptoms.

\section{Traditional markers of cardiovascular risk}

Compared with patients with active acromegaly, patients in remission had higherLDLand triglyceridelevels. Nodifference was identified in relation to mean age, gender distribution, body composition, glucose profile and prevalence of other cardiovascular risk factors. Patients with active disease had significantly higher BMI, LDL cholesterol and prevalence of diabetes/IGT compared with controls. Patients with disease remission also demonstrated a less favourable metabolic and cardiovascular risk profile compared with controls, due to higher BMI, waist/hip ratio (WHR), fat mass, triglycerides and prevalence of hypertension and dyslipidaemia. Results are summarised in Table 2.

\section{Clot structure analysis - assessing the impact of disease activity}

Following adjustment for BMI, patients with active acromegaly had shorter lag time compared with those 
Table 1 Summary of the acromegaly-related past medical history (biochemical results, disease status at the time of the study, previous therapeutic interventions and pituitary-related outcomes) for patients with active disease $(n=22)$ and disease remission $(n=18)$.

Age (years)

Male/Female

Current GH $(\mu \mathrm{g} / \mathrm{L})$

Current IGF-1 (\% ULN)

Mean age at diagnosis of acromegaly (years)

Estimated age at onset of symptoms (years)

Duration of active disease (years)

Duration of disease remission (years)

$\mathrm{GH}$ at diagnosis $(\mu \mathrm{g} / \mathrm{L})$

IGF-1 at diagnosis (\% ULN)

Trans-sphenoidal surgery

Pituitary radiotherapy

Medical therapy

Hypopituitarism

LH/FSH deficiency

ACTH deficiency

TSH deficiency

ADH deficiency

\begin{tabular}{c}
$\begin{array}{c}\text { Patients with active acromegaly } \\
(n=22)\end{array}$ \\
\hline $51 \pm 13$ \\
$13 / 9$ \\
$2.6(0.8-3.5)$ \\
$131.7(106.9-212.2)$ \\
$40.6 \pm 12.8$ \\
$33.7 \pm 12.5$ \\
$11.8(8.25-23.5)$ \\
$0(0-2.0)$ \\
$19.4(5.9-33.3)$ \\
$307(158.7-413.5)$ \\
$19(86.4 \%)$ \\
$13(59.1 \%)$ \\
$14(63.6 \%)$ \\
$6(27.3 \%)$ \\
$9(40.9 \%)$ \\
$5(22.7 \%)$ \\
$0(0 \%)$
\end{tabular}

\begin{tabular}{c}
\hline P-value \\
\hline 0.3 \\
0.545 \\
$<\mathbf{0 . 0 0 1}$ \\
$<\mathbf{0 . 0 0 1}$ \\
0.93 \\
0.9 \\
0.11 \\
$<\mathbf{0 . 0 0 1}$ \\
0.34 \\
0.85 \\
1.00 \\
0.42 \\
0.37 \\
\\
0.66 \\
0.92 \\
0.7 \\
0.08 \\
\hline
\end{tabular}

in remission $(515.6 \pm 15.1$ vs $570.2 \pm 16.8 \mathrm{~s}, P=0.02)$; however, clot MA was similar in the two groups (Group 1: $0.41 \pm 0.03$ arbitrary units (AU); Group 2: $0.35 \pm 0.03$ AU, $P=0.18)$. Additionally, no statistical difference was found either in 50\% lysis time (Group 1: $25.5 \pm 4.3 \mathrm{~min}$; Group 2: $33.2 \pm 4.8 \mathrm{~min} ; P=0.24$ ) or lysis areas (Group 1: $849.7 \pm 130.9$ AU; Group 2: 837.9 \pm 144.8 AU, $P=0.95)$. Results are summarised in Table 3.

When patients from each subgroup were compared with controls and following adjustment for BMI, patients with active disease had significantly higher clot MA $(0.39 \pm 0.02$ vs $0.33 \pm 0.01 \mathrm{AU}, P=0.03)$. No difference was found in the lag time for clot formation; 50\% lysis time and lysis area. In contrast, there was no difference in maximum clot MA between patients with disease remission and controls. There was a trend towards longer lysis time and larger lysis area for patients with disease remission compared with controls, although the difference did not reach statistical significance. A summary of the results can be found in Table 3 .

\section{Coagulation proteins and CRP plasma levels}

Following adjustment for BMI, no difference in fibrinogen, PAI-1 and CRP was observed between patients with active acromegaly and those in remission (Table 3 ).

Patients with active disease had significantly higher fibrinogen concentrations compared with controls $(3.8 \pm 0.2$ vs $2.6 \pm 0.2 \mathrm{mg} / \mathrm{mL}$, respectively, $P<0.001)$, which is in keeping with the higher clot MA observed in the patient group. PAI-1 levels were similar in the two study groups, which is also consistent with the lysis data. No difference in plasma CRP was observed. Patients with disease remission also had higher fibrinogen levels compared with controls $(3.25 \pm 0.2$ vs $2.6 \pm 0.2 \mathrm{mg} / \mathrm{mL}$, $P=0.02)$; however, the difference was greater for patients with active disease. No difference in PAI-1 and CRP was observed between patients with disease remission and controls (Table 3).

\section{Correlations - patient group}

Multiple linear regression analysis was performed as described in the methodology. Lag time was negatively associated with fibrinogen (co-efficient $-17.8, P=0.012$ ); current $\mathrm{GH}$ values (co-efficient $-11.5, P=0.021$ ) and smoking (co-efficient $-32.5, P=0.036$ ).

Clot MA was positively associated with fibrinogen levels (co-efficient 0.06, $P<0.001$ ); BMI (co-efficient 0.008, $P=0.044$ ); total fat mass (co-efficient 0.004, $P=0.041$ ) and summative skinfold thickness (co-efficient 0.002, $P=0.048)$. No associations were found with acromegalyrelated factors.

Lysis time was positively correlated with PAI-1 levels (coefficient 1.1, $P<0.001$ ); diabetes (co-efficient 10.9, $P=0.03$ ) and summative skinfold thickness (co-efficient 0.28, $P=0.043$ ), with a trend for BMI (co-efficient 1.08, $P=0.07)$. 


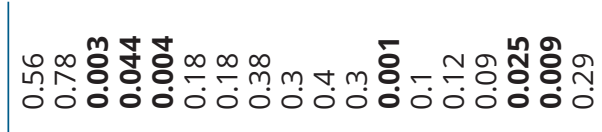

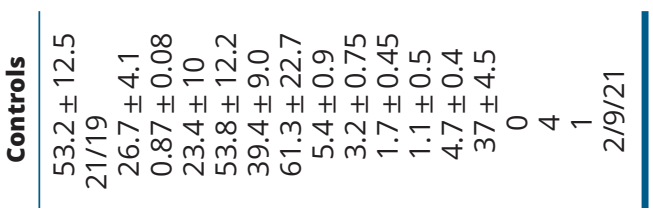

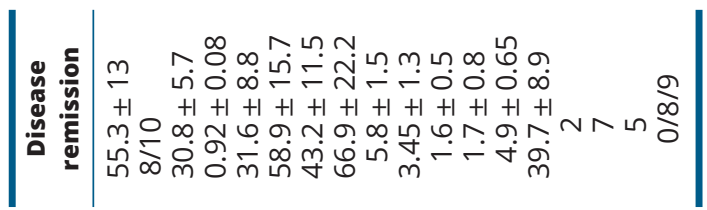

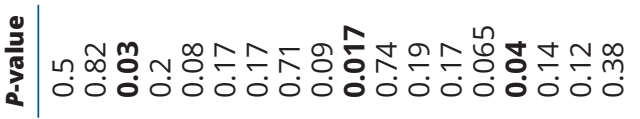

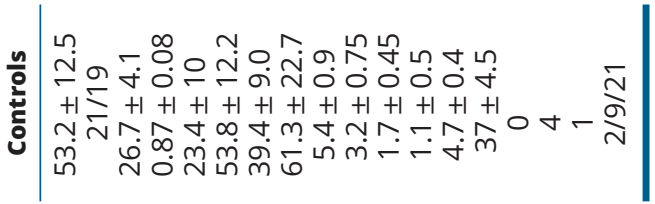

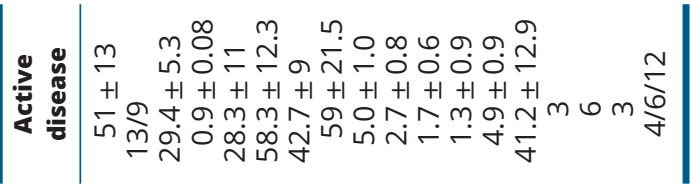
疍|

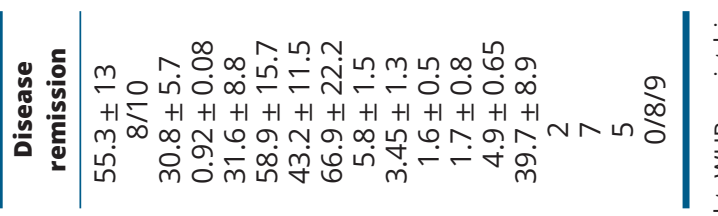

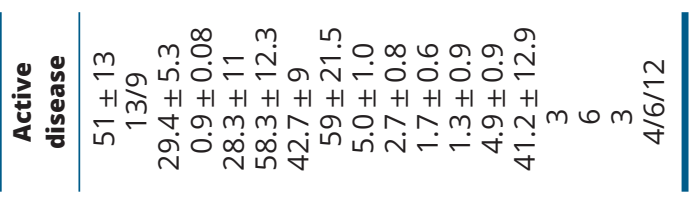
$\frac{\mathrm{O}}{\mathrm{D}}$ 당

음 竞 응 을 ¿ ते ๑

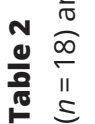

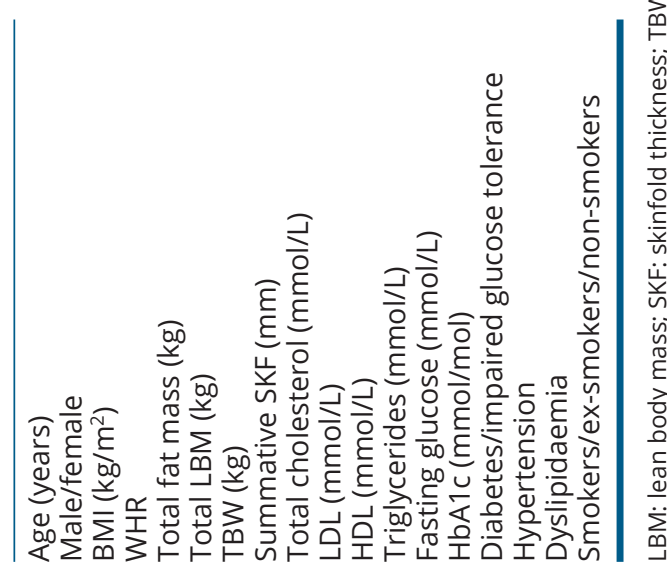

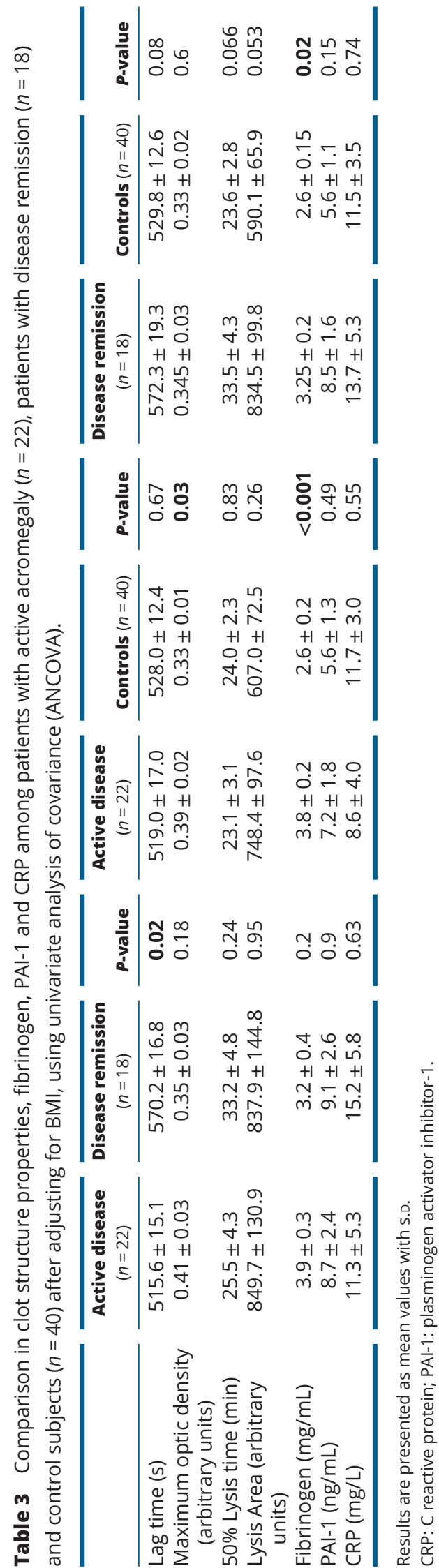




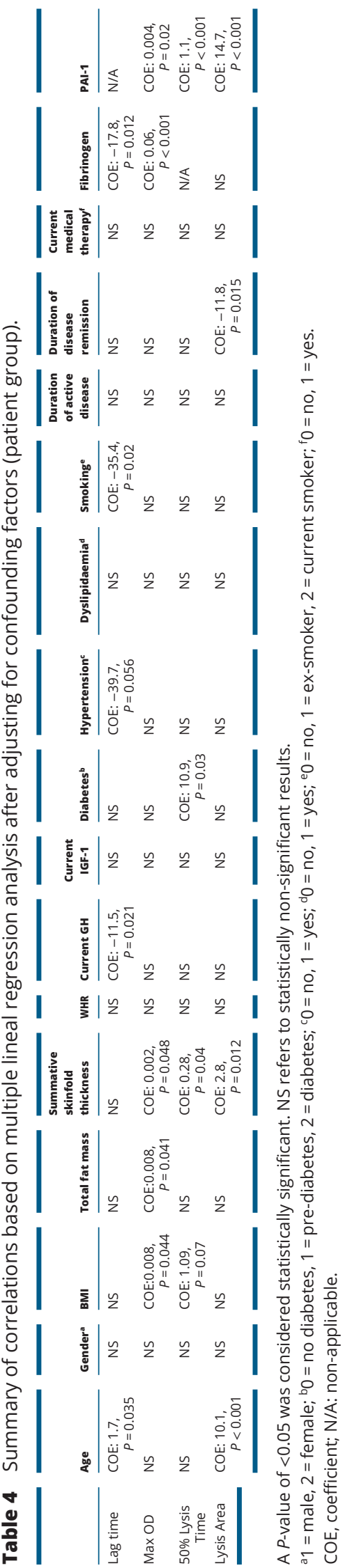

Lysis area was positively correlated with PAI-1 levels (coefficient 14.7, $P<0.001$ ); older patient's age (coefficient 10.1, $P<0.001)$ and summative skinfold thickness (coefficient 2.8, $P=0.012$ ). There was also a negative correlation between lysis area and duration of remission of acromegaly (coefficient $-11.8, P=0.015$ ).

A positive correlation was found between serum fibrinogen and duration of active disease (co-efficient 0.06, $P=0.034$ ) and smoking (co-efficient $0.9, P=0.038$ ), whereas PAI-1 was positively associated with BMI (coefficient 0.87, $P=0.039$ ). Table 4 summarises the previously mentioned results.

\section{Correlations - control group}

A negative correlation between lag time and WHR was found (co-efficient -549.4, P=0.015). Clot MA was positively correlated with fibrinogen (co-efficient $0.167, P<0.001)$ and lysis time and lysis area with PAI-1 levels (co-efficient 1.423, $P=0.01$; and 19.39, $P=0.02$ respectively). Total fat mass, WHR and summative skinfold thickness were positively correlated with fibrinogen (coefficient 0.013, $P=0.03 ; 2.074, P=0.025$; and 0.01, $P=0.005$, respectively), but not directly with clot MA. Finally, a positive correlation was found between PAI-1 and HbA1c (co-efficient 0.43, P=0.02); PAI-1 and WHR (co-efficient $32.5, P=0.006$ ); and PAI-1 and total fat mass (co-efficient $0.17, P=0.038$ ).

\section{Laser scanning confocal microscopy (LSCM)}

Patients with active acromegaly were found to have a more dense fibrin network, not only compared with controls (mean number of fibrin fibres/100 $\mu \mathrm{m} \mathrm{30.4 \pm 1.3}$ vs $24.1 \pm 1.2, P=0.004)$, but also with patients with disease remission (mean fibrin fibres/100 $\mu \mathrm{m} 30.4 \pm 1.3$ vs $25.3 \pm 0.9, P=0.005$; Fig. 1 ). There was no difference in the density of the fibrin network between patients in remission and controls (mean fibrin fibres/100 $\mu \mathrm{m}$ $25.3 \pm 0.9$ vs $24.1 \pm 1.2$, respectively, $P=0.24$; Fig. 1 ). These findings are consistent with the turbidimetric assay data previously presented.

\section{Discussion}

Patients with acromegaly have increased cardiovascular mortality $(3,4,5,6,7)$ and an increased thrombotic milieu has been proposed (11, 12, 14, 27). However, previous studies have only examined plasma levels or 

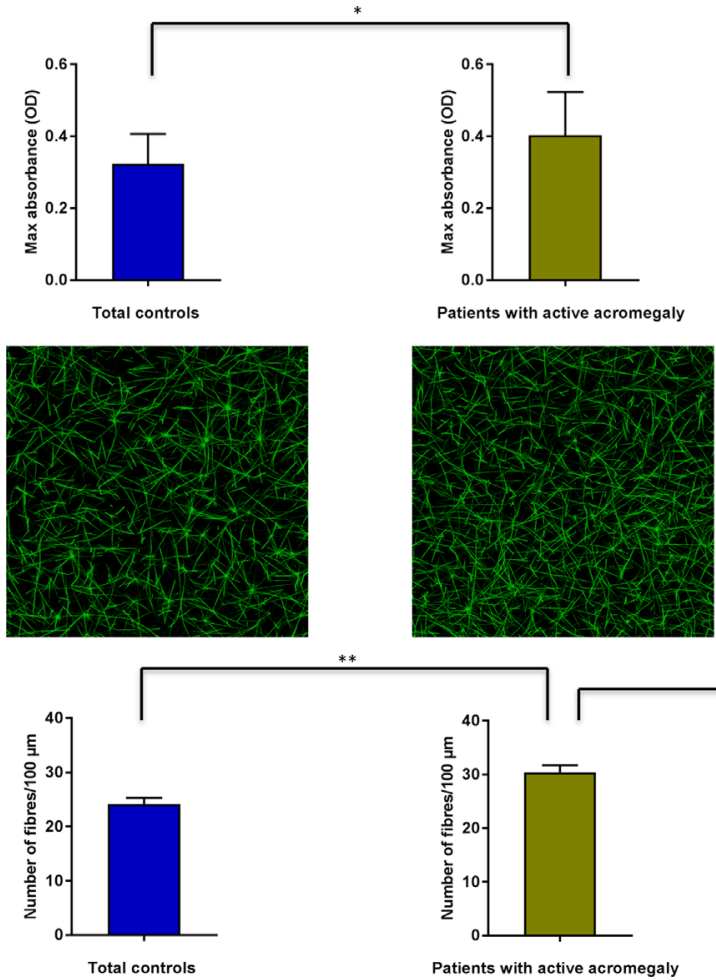

activity of clotting and fibrinolytic factors, whereas this study has the advantage of a more global assessment of clot formation and clot lysis. This pilot study is the first to show that patients with active acromegaly have more compact clots compared with controls matched for age and gender, based on an ex vivo clot structure analysis and after adjustment for BMI. Laser scanning confocal microscopy suggested that the difference in the fibrin clot density is more prominent in the group of patients with active disease, with the groups of patients with longterm disease remission and controls being essentially indistinguishable.

Similar to previous studies $(8,9,10,11,12,13,14$, $15)$, we were able to demonstrate higher fibrinogen levels among patients with active acromegaly compared with controls, which translated into higher clot MA. Despite this, lysis time was not statistically different and neither were PAI-1 levels. Overall, the results suggest that patients with active acromegaly have increased thrombotic potential with increased clot density; however, the fibrinolytic system does not seem to be significantly

\section{Figure 1}

The middle panel shows the fibrin network of clots formed ex vivo from pooled plasmas of (1.) all controls $(n=40)$; (2.) patients with active disease $(n=22)$ and (3.) patients with disease remission ( $n=18)$, obtained by laser scanning confocal microscopy, in conjunction with clots maximum optic density (top panel), as calculated by turbidimetric assay and number of fibres per $100 \mu \mathrm{m}$ (bottom panel) incorporated in the clot structure. A higher density of clot fibrin network was observed in patients with active acromegaly compared with controls, which was also supported by the higher clot maximum optic density ( ${ }^{*} P=0.004$ ) and the higher number of fibres $/ 100 \mu \mathrm{m}$ ( $* * P=0.004)$. Patients with active disease also had higher number of fibres $/ 100 \mu \mathrm{m}$ compared with patients with disease remission ( $* * * P=0.005)$. In contrast, there was no difference in the maximum clot optic density and number of fibres/100 $\mu \mathrm{m}$ between patients with disease remission and controls. affected. Following adjustment for BMI, patients with disease remission were also found to have higher fibrinogen levels compared with controls; however, this did not lead in significant differences in clot MA or in the clot fibrin network density as visualised by LSCM. This suggests that the increased thrombotic potential in acromegaly may at least be partially reversed following successful treatment and biochemical disease control. The results of LSCM are in support with the above observations, which showed significantly higher number of fibrin fibres per $100 \mu \mathrm{m}$ in patients with active acromegaly compared with those in remission, suggesting the presence of increased fibrin network density in the former patient group.

Investigating factors that may influence clot structure properties in patients with acromegaly, the adverse metabolic profile was associated with increased thrombotic potential in these patients. Elevated BMI, total fat mass, summative skinfold thickness and diagnosis of diabetes/ IGT were all independent risk factors for adverse clot formation and lysis properties. Similar associations were also found in the control group and although the adverse 
metabolic profile was not directly related to unfavourable clot formation and lysis properties, it was associated with higher fibrinogen and PAI-1 levels.

It is well-recognized that acromegaly is associated with a variety of metabolic complications including diabetes/IGT, hypertension and disorders of lipid metabolism (28). This was also evident by the results of this study. Considering the effect of the adverse metabolic profile, body composition and diabetes on clot formation and lysis properties, it is essential that patients with acromegaly are screened and appropriately treated for these complications, but even more importantly that acromegaly is diagnosed early in the disease course and treated successfully, in order to minimise duration of active disease and prevent complications from arising. Notably, in our study, patients with disease remission continued to exhibit an adverse profile of body composition and cardiovascular risk factors, as evident by the higher WHR, fat mass, triglycerides and rates of hypertension and dyslipidaemia, and despite no difference in clot fibrin network density, this may account for the higher fibrinogen levels and the trend towards more prolonged lysis time and larger lysis area compared with controls, following adjustment for BMI.

However, adverse body composition and diabetes are not the only factors responsible for the negative impact on clot formation properties in patients with acromegaly. Based on multiple linear regression and following adjustment for for age, gender and metabolic parameters; longer duration of active disease was associated with higher fibrinogen levels; shorter duration of disease remission was associated with larger lysis area; and higher GH levels at the time of the study were associated with shorter lag time for clot formation, suggestin that disease activity adversely affects the thrombotic potential in patients with acromegaly, independently of the metabolic complications. Additionally, when comparing patients with active acromegaly with patients in remission, body composition and prevalence of metabolic complications were similar between the two groups, as were mean age and gender distribution. Despite the previously mentioned similarities, LSCM showed more compact clots with higher concentration of fibrin fibres, which further strengthens the hypothesis that active acromegaly independently increases the thrombotic potential in these patients. This is consistent with previous all cause mortality and cardiovascular mortality data, which have shown increased SMR in patients with active acromegaly, but not in those with disease remission $(1,2,3,4)$.
Limitations to the study include the relatively small number of patients in each disease status subgroup, which may have led to a type II statistical error, when clot structure properties were compared between patients with active acromegaly and patients with disease remission. A significant proportion of the initially screened patients for this study (21 of 91 screened, $23.1 \%$ ) were excluded, as they were on treatment with antiplatelet or anticoagulant agents due to established cardiovascular morbidity. Therefore, by excluding this high-risk subgroup of acromegalic patients, it is possible that the study has underestimated the severity of the clot structure abnormalities and the effect of the disease on the increased thrombotic potential of patients with acromegaly.

Further prospective studies are required to fully elucidate the effect of acromegaly on clot structure properties. These studies should aim to assess clot formation and lysis in patients with active acromegaly before and after treatment and biochemical disease control, in a multi-centre setting, and also link clot structure properties with cardiovascular outcomes. In addition to fibrinogen levels, a number of proteins have been detected in the fibrin network that may alter properties and resistance to lysis including fibronectin, $\alpha_{2}$-antiplasmin, complement C3, histidine-rich glycoprotein and apolipoproteins (29). These warrant further investigation to establish the exact mechanisms for altered clot structure in individuals with acromegaly.

In conclusion, this pilot study provides new evidence that patients with active acromegaly have abnormal clot structure properties, particularly with regards to clot formation, with higher maximum clot density and more compact clots. This may represent one mechanism for the increased cardiovascular risk observed in patients with acromegaly, particularly during active disease. The effect of acromegaly on the abnormal clot structure properties is likely multifactorial, with the adverse metabolic profile observed in these patients, as well as disease activity being associated with increased thrombotic potential.

\section{Declaration of interest}

The authors declare that there is no conflict of interest that could be perceived as prejudicing the impartiality of this study.

\section{Funding}

This research did not receive any specific grant from funding agencies in the public, commercial or not-for-profit sectors. 


\section{Acknowledgments}

The authors would like to thank Dr Julian Barth, Consultant Biochemist, and Dr Stephen Gibbons, Principal Clinical Biochemist, Department of Biochemistry, Leeds Teaching Hospitals NHS Trust, for providing with the relevant details on hormone and other biochemical assays.

\section{References}

1 Dekkers OM, Biermasz NR, Pereira AM, Romijn JA \&

Vandenbroucke JP. Mortality in acromegaly: a metaanalysis. Journal of Clinical Endocrinology and Metabolism 200893 61-67. (https://doi. org/10.1210/jc.2007-1191)

2 Holdaway IM, Bolland MJ \& Gamble GD. A meta-analysis of the effect of lowering serum levels of GH and IGF-1 on mortality in acromegaly. European Journal of Endocrinology 2008159 89-95. (https://doi.org/10.1530/EJE-08-0267)

3 Bolfi F, Neves AF, Boguszewski CL \& Nunes-Nogueira VS. Mortality in acromegaly decreased in the last decade: a systematic review and meta-analysis. European Journal of Endocrinology 2018179 59-71. (https://doi.org/10.1530/EJE-18-0255)

4 Orme SM, McNally RJ, Cartwright RA \& Belchetz PE. Mortality and cancer incidence in acromegaly: a retrospective cohort study. United Kingdom Acromegaly Study Group. Journal of Clinical Endocrinology and Metabolism 199883 2730-2734. (https://doi.org/10.1210/ jcem.83.8.5007)

5 Holdaway IM, Rajasoorya RC \& Gamble GD. Factors influencing mortality in acromegaly. Journal of Clinical Endocrinology and Metabolism 200489 667-674. (https://doi.org/10.1210/jc.2003031199)

6 Sherlock M, Reulen RC, Alonso AA, Ayuk J, Clayton RN, Sheppard MC, Hawkins MM, Bates AS \& Stewart PM. ACTH deficiency, higher doses of hydrocortisone replacement and radiotherapy are independent predictors of mortality in patients with acromegaly. Journal of Clinical Endocrinology and Metabolism 200994 4216-4223. (https://doi.org/10.1210/jc.2009-1097)

7 Esposito D, Ragnarsson O, Granfeldt D, Marlow T, Johannsson G \& Olsson DS. Decreasing mortality and changes in treatment patterns in patients with acromegaly from a nationwide study. European Journal of Endocrinology 2018178 459-469. (https://doi.org/10.1530/ EJE-18-0015)

8 Landin-Wilhelmsen K, Tengborn L, Wilhelmsen L \& Bengtsson BA. Elevated fibrinogen levels decrease following treatment of acromegaly. Clinical Endocrinology 199746 69-74. (https://doi. org/10.1046/j.1365-2265.1997.d01-1743.x)

9 Sartorio A, Cattaneo M, Bucciarelli P, Bottasso B, Porretti S, Epaminonda P, Faglia G \& Arosio M. Alterations of haemostatic and fibrinolytic markers in adults patients with growth hormone deficiency and with acromegaly. Experimental and Clinical Endocrinology and Diabetes 2000108 486-492. (https://doi. org/10.1055/s-2000-8145)

10 Colao A, Spinelli L, Cuocolo A, Spiezia S, Pivonello R, di Somma C, Bonaduce D, Salvatore M \& Lombardi G. Cardiovascular consequences of early-onset growth hormone excess. Journal of Clinical Endocrinology and Metabolism 200287 3097-3104. (https:// doi.org/10.1210/jcem.87.7.8573)

11 Vilar L, Naves LA, Costa SS, Abdalla LF, Coelho CE \& Casulari LA. Increase of classic and nonclassic cardiovascular risk factors in patients with acromegaly. Endocrine Practice 200713 363-372. (https://doi.org/10.4158/EP.13.4.363)

12 Erem C, Nuhoglu I, Kocak M, Yilmaz M, Sipahi ST, Ucuncu O \& Ersoz HO. Blood coagulation and fibrinolysis in patients with acromegaly: increased plasminogen activator inhibitor-1 (PAI-1), decreased tissue factor pathway inhibitor (TFPI), and an inverse correlation between growth hormone and TFPI. Endocrine 200833 270-276. (https://doi.org/10.1007/s12020-008-9088-4)

13 Kaluzny M, Bolanowski M, Daroszewski J \& Szuba A. The role of fibrinogen and CRP in cardiovascular risk in patients with acromegaly. Endokrynologia Polska 201061 83-88.

14 Colak A, Yilmaz H, Temel Y, Demirpence M, Simsek N, Karademirci İ, Bozkurt U \& Yasar E. Coagulation parameters and platelet function analysis in patients with acromegaly. Journal of Endocrinological Investigation 201639 97-101. (https://doi.org/10.1007/s40618-0150300-0)

15 Amado A, Araújo F \& Carvalho D. Cardiovascular risk factors in acromegaly: what's the impact of disease control? Experimental and Clinical Endocrinology and Diabetes 2018126 505-512. (https://doi. org/10.1055/s-0043-124668)

16 Wildbrett J, Hanefeld M, Fücker K, Pinzer T, Bergmann S, Siegert G \& Breidert M. Anomalies of lipoprotein pattern and fibrinolysis in acromegalic patients: relation to growth hormone levels and insulin-like growth factor I. Experimental and Clinical Endocrinology and Diabetes 1997105 331-335. (https://doi. org/10.1055/s-0029-1211774)

17 Mills JD, Ariëns RA, Mansfield MW \& Grant PJ. Altered fibrin clot structure in healthy relatives of patients with premature coronary artery disease. Circulation 2002106 1938-1942. (https://doi. org/10.1161/01.cir.0000033221.73082.06)

18 Collet JP, Allali Y, Lesty C, Tanguy ML, Silvain J, Ankri A, Blanchet B, Dumaine R, Gianetti J, Payot L et al. Altered fibrin architecture is associated with hypofibrinolysis and premature coronary atherothrombosis. Arteriosclerosis, Thrombosis, and Vascular Biology 200626 2567-2573. (https://doi.org/10.1161/01. ATV.0000241589.52950.4c)

19 Sumaya W, Wallentin L, James SK, Siegbahn A, Gabrysch K, Bertilsson M, Himmelmann A, Ajjan RA \& Storey RF. Fibrin clot properties independently predict clinical outcome following acute coronary syndrome: a Plato substudy. European Heart Journal 201839 1078-1085. (https://doi.org/10.1093/eurheartj/ehy013)

20 Katznelson L, Laws Jr ER, Melmed S, Molitch ME, Murad MH, Utz A, Wass JA \& Endocrine Society. Acromegaly: an endocrine society clinical practice guideline. Journal of Clinical Endocrinology and Metabolism 201499 3933-3951. (https://doi.org/10.1210/jc.20142700)

21 Libby P \& Ridker PM. Inflammation and atherosclerosis: role of C-reactive protein in risk assessment. American Journal of Medicine 2004116 (Supplement 6A) 9S-16S. (https://doi.org/10.1016/j. amjmed.2004.02.006)

22 Carter AM, Cymbalista CM, Spector TD, Grant PJ \& EuroCLOT Investigators. Heritability of clot formation, morphology, and lysis: the EuroCLOT study. Arteriosclerosis, Thrombosis, and Vascular Biology 200727 2783-2789. (https://doi.org/10.1161/ATVBAHA.107.153221)

23 Cooper AV, Standeven KF \& Ariëns RA. Fibrinogen gamma-chain splice variant gamma' alters fibrin formation and structure. Blood 2003102 535-540. (https://doi.org/10.1182/blood-2002-10-3150)

24 Undas A, Kolarz M, Kopeć G \& Tracz W. Altered fibrin clot properties in patients on long-term haemodialysis: relation to cardiovascular mortality. Nephrology, Dialysis, Transplantation 200823 2010-2015. (https://doi.org/10.1093/ndt/gfm884)

25 Undas A, Szuldrzynski K, Stepien E, Zalewski J, Godlewski J, Tracz W, Pasowicz M \& Zmudka K. Reduced clot permeability and susceptibility to lysis in patients with acute coronary syndrome: effects of inflammation and oxidative stress. Atherosclerosis 2008196 551-557. (https://doi.org/10.1016/j.atherosclerosis.2007.05.028)

26 Ajjan R, Lim BC, Standeven KF, Harrand R, Dolling S, Phoenix F, Greaves R, Abou-Saleh RH, Connell S, Smith DA et al. Common variation in the C-terminal region of the fibrinogen $\beta$-chain: effects on fibrin structure, fibrinolysis and clot rigidity. Blood $2008 \mathbf{1 1 1}$ 643-650. (https://doi.org/10.1182/blood-2007-05-091231) 
27 Kyriakakis N, Lynch J, Ajjan R \& Murray RD. The effects of pituitary and thyroid disorders on haemostasis: potential clinical implications. Clinical Endocrinology 201684 473-484. (https://doi.org/10.1111/ cen.12767)

28 Colao A, Ferone D, Marzullo P \& Lombardi G. Systemic complications of acromegaly: epidemiology, pathogenesis, and management. Endocrine Reviews 200425 102-152. (https://doi. org/10.1210/er.2002-0022)

29 Ząbczyk M, Stachowicz A, Natorska J, Olszanecki R, Wiśniewski JR \& Undas A. Plasma fibrin clot proteomics in healthy subjects: relation to clot permeability and lysis time. Journal of Proteomics 2019208 103487. (https://doi.org/10.1016/j.jprot.2019.103487)

Received 13 October 2019

Revised version received 11 March 2020

Accepted 19 March 2020 